\title{
OPEN Forensic significance of intracardiac heme oxygenase- 1 expression in acute myocardial ischemia
}

\author{
Yumi Kuninaka ${ }^{1,5}$, Yuko Ishida ${ }^{1,5}$, Mizuho Nosaka ${ }^{1}$, Akiko Ishigami ${ }^{1}$, Akira Taruya ${ }^{2}$, \\ Emi Shimada ${ }^{1}$, Akihiko Kimura ${ }^{1}$, Hiroki Yamamoto ${ }^{1}$, Mitsunori Ozaki ${ }^{3}$, Fukumi Furukawa ${ }^{1,4}$ \& \\ Toshikazu Kondo ${ }^{1 凶}$
}

Heme oxygenase-1 (HO-1), an inducible stress-response protein, exerts anti-oxidant and antiapoptotic effects. However, its significance in forensic diagnosis of acute ischemic heart diseases (AIHD) such as myocardial infarction (MI) is still unknown. We examined the immunohistochemical expression of HO-1 in the heart samples to discuss their forensic significance to determine acute cardiac ischemia. The heart samples were obtained from 23 AIHD cases and 33 non-AIHD cases as controls. HO-1 positive signals in cardiomyocyte nuclear were detected in $78.2 \%$ of AlHD cases, however, that were detected in only $24.2 \%$ control cases with statistical difference between AIHD and non-AIHD groups. In contrast to HO-1 protein expression, there was no significant difference in the appearance of myoglobin pallor regions and leukocyte infiltration in the hearts between AIHD and non-AIHD groups. From the viewpoints of forensic pathology, intracardiac HO-1 expression would be considered a valuable marker to diagnose AIHD as the cause of death.

Ischemic heart disease is the leading cause of death in the worldwide and is the most common cause of sudden cardiac death $(S C D)^{1,2}$. In the cases of SCD, the postmortem diagnosis of acute myocardial ischemia is an important issue for both clinical physicians and forensic pathologists if death occurs within a short period of time after the onset of ischemic heart attack.

Myocardial infarction (MI), pathologically defined as myocardial cell death due to prolonged ischemia, is most representative acute ischemic heart disease (AIHD). After the onset of myocardial ischemia, histological cell death does not occur immediately, and it takes a finite period of time to develop-as little as $20 \mathrm{~min}$, or less in some animal models ${ }^{3-5}$. Depending on the sensitivity of cardiomyocytes, macroscopic or microscopic postmortem examination takes several hours to identify myocardial necrosis. Thus, the evaluation of early myocardial damage using routine histological examination such as HE staining is only possible in death cases at least several hours passed after the onset of ischemic damage.

To identify early signs of cardiac ischemia, histochemical techniques have been proposed ${ }^{6-12}$. However, these methods are not suitable for routine use, and their specificity and sensitivity are controversial because positive results may be sometimes associated with agonal factors and postmortem changes ${ }^{13,14}$. Recent works have immunohistochemically investigated, some markers such as fibronectin, C5b-9 and myoglobin which accumulate in or leak from cardiomyocytes after ischemia. However, these molecules almost failed to be detected in the very early phase of myocardial ischemia ${ }^{5,15-18}$.

Heme oxygenase-1 (HO-1, HSP32) is a vital heme degradative enzyme and inducible stress protein ${ }^{19-21}$. Bilirubin and carbon monoxide $(\mathrm{CO})$ that are reaction products of $\mathrm{HO}-1$ are protective against ischemia-induced injury such as myocardial infarction, ischemia-reperfusion injury, and post-infarct structural remodeling ${ }^{22-24}$. Oxidative stress associated with over-vasoconstriction is the major pathogenic mechanism in the various heart pathologies. Nuclear translocation of HO-1 induced by oxidative stress resulted in the promoted interaction of $\mathrm{HO}-1$ with the redox-sensitive transcription factor $\mathrm{Nrf} 2{ }^{25}$. The observations described previously suggest that HO- 1 had a role in the pathogenesis of AIHD. However, the knowledge of their involvement in AIHD and their significance in forensic diagnose are insufficient. Therefore, in the present study, we examined intracardiac

\footnotetext{
${ }^{1}$ Department of Forensic Medicine, Wakayama Medical University, 811-1 Kimiidera, Wakayama 641-8509, Japan. ${ }^{2}$ Department of Cardiovascular Medicine, Wakayama Medical University, 811-1 Kimiidera, Wakayama 641-8509, Japan. ${ }^{3}$ Department of Neurological Surgery, Wakayama Medical University, 811-1 Kimiidera, Wakayama 641-8509, Japan. ${ }^{4} T a k a t s u k i$ Red Cross Hospital, 1-1-1 Abuno, Takatsuki-shi, Osaka 569-1096, Japan. ${ }^{5}$ These authors contributed equally: Yumi Kuninaka and Yuko Ishida. ${ }^{\square}$ email: iyuko@ wakayama-med.ac.jp; kondot@wakayama-med.ac.jp
} 

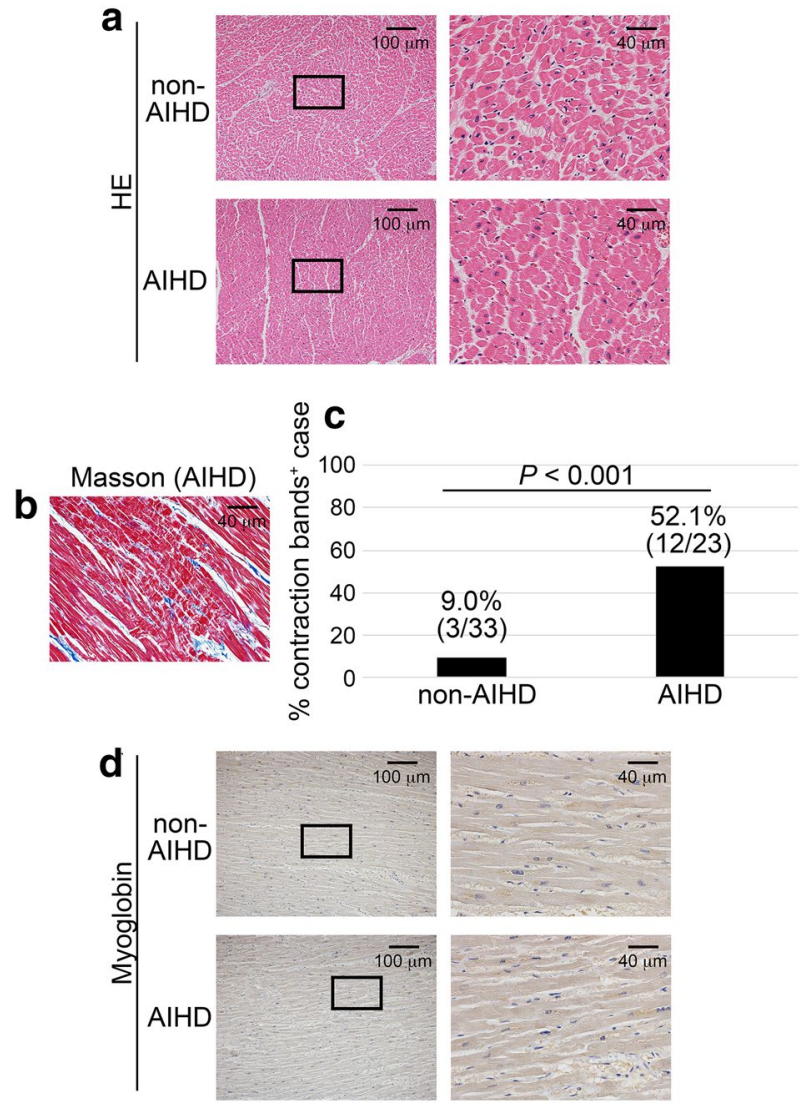

Figure 1. Histopathological and immunohistochemical analysis of the human hearts. (a) HE staining. Representative results from non-AIHD (Drowning) and AIHD groups were shown here. (b) Contraction bands on the heart of AIHD group (Masson trichrome staining). (c) Percentage of cases where contraction bands were present. (d) Immunohistochemical analysis by using anti-myoglobin. Representative results from non-AIHD (Drowning) and AIHD groups were shown here.

HO-1 protein expression in forensic autopsy samples and discussed its availability as a potential indicator for postmortem diagnosis of AIHD.

\section{Results}

Histopathological and immunohistochemical analyses in autopsy samples. Histopathologically, HE staining showed that there was no specific histological sign of MI in both AIHD and non-AIHD groups (Fig. 1a and Supplemental Fig. 1). However, contraction bands could be confirmed by Masson trichrome staining in some of AIHD groups (Fig. 1b), and there was a significant difference of percentage of the case where contraction band existed evidently between AIHD and non-AIHD groups (52.1\% vs. 9.0\%, Fig. 1c). Moreover, the immunohistochemical staining of myoglobin revealed no differences in the appearance of myoglobin pallor regions between AIHD and non-AIHD groups (Fig. 1d and Supplemental Fig. 2).

Intracardiac leukocyte infiltration. Intracardiac leukocyte recruitment is also one of the hallmarks in AIHD. Thus, we examined the degrees of intracardiac leukocyte recruitment. Subsequently, immunohistochemical analyses demonstrated that few $\mathrm{MPO}^{+}$neutrophils (Fig. 2a and Supplemental Fig. 3) and macrophages (Fig. 2b and Supplemental Fig. 4) were present in the control hearts. In AIHD hearts, neutrophils and macrophages were observed to a similar extent as non-AIHD (Fig. 2). These observations implied that the evaluation of leukocyte recruitment would not be powerful clue for the diagnosis of early phase of AIHD.

Enhanced intracardiac HO-1 expression in AIHD group. We could observe intracardiac HO-1 expression in both groups. The immunohistochemical expression of HO-1 was more intense and diffuses in the nuclei of cardiomyocytes in AIHD group, compared with non-AIHD group ones (Fig. 3a and Supplemental Fig. 5). The comparison between AIHD and non-AIHD groups showed significant difference of the number of HO-1 expressed case (Fig. 3b). There were no significant differences on age, gender, or PMI for HO-1 protein expressions (Fig. 4). 

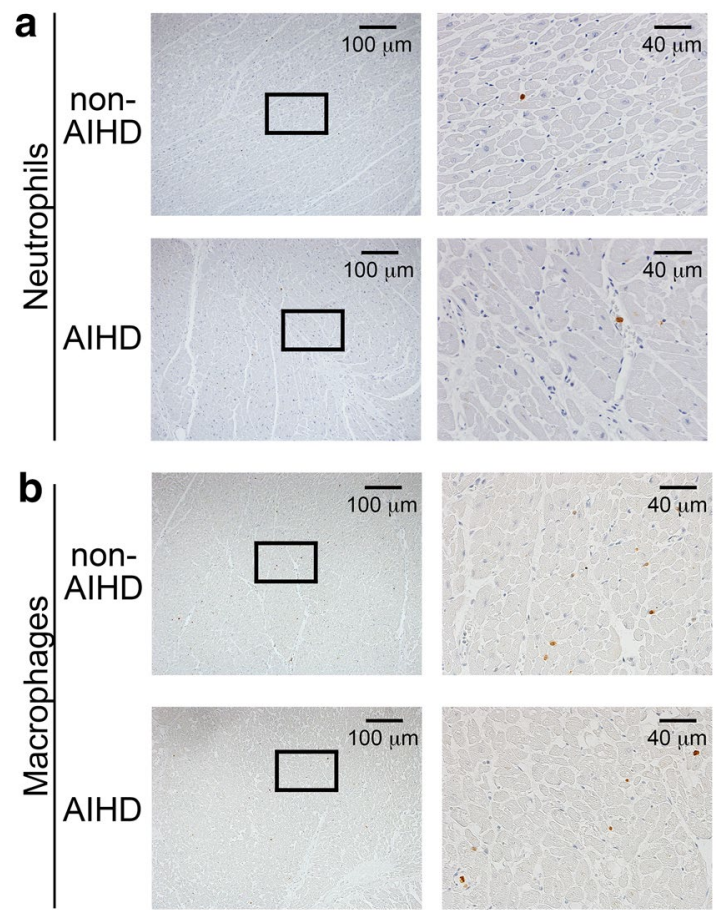

Figure 2. Detection of leukocyte infiltration in the non-AIHD and AIHD hearts. (a,b) Immunohistochemical analysis by using anti-MPO for neutrophils (a) and anti-Macrophage Marker for macrophages (b).

Representative results from non-AIHD (Drowning) and AIHD groups were shown here.

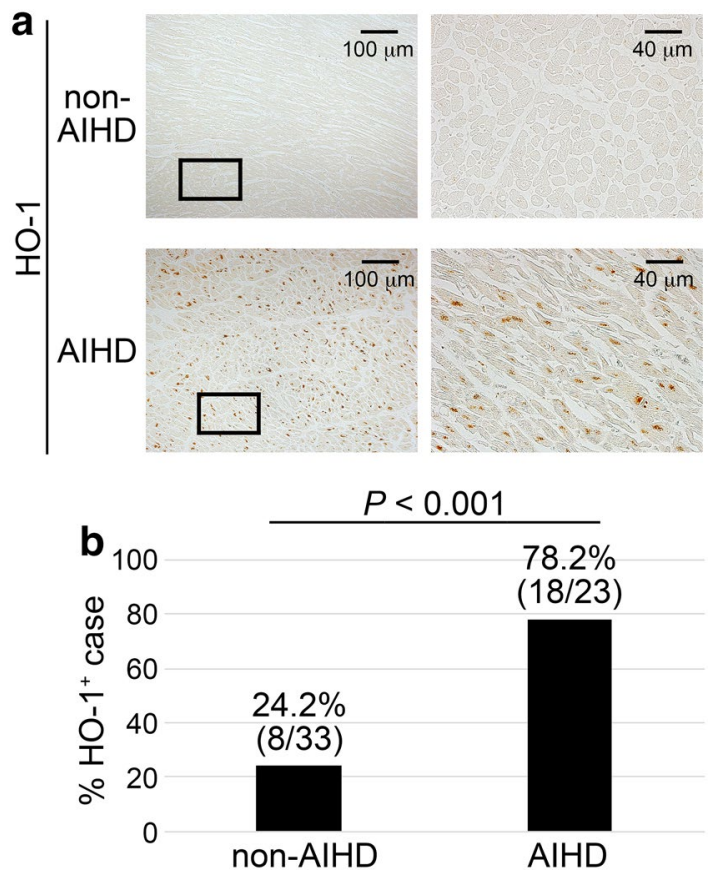

Figure 3. The expression of HO-1 on the human hearts. (a) Immunohistochemical analysis by using anti-HO-1. Representative results from the hearts of non-AIHD (Drowning) and AIHD groups were shown here. (b) Percentage of cases where HO-1-positive nuclei of cardiomyocytes were exist.

\section{Discussion}

The post-mortem diagnosis of SCD can be difficult if it is based only on routine investigation as heart macroscopic findings and HE analyses. As a method for detecting myocardial ischemia, visualization of molecular change by immunohistochemical examination has been proposed, but its application depends on the availability of a specific and sensitive marker ${ }^{15,26,27}$. Until now, several lines of accumulating evidence implied the possibility 

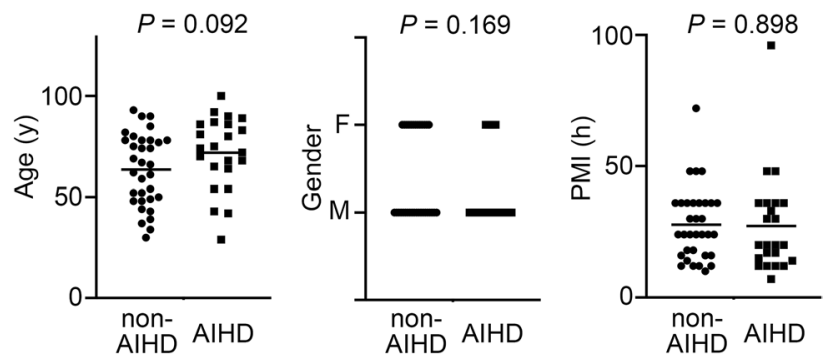

Figure 4. The relation between age, gender, or PMI, and non-AIHD and AIHD groups. These results were obtained with Spearman's correction coefficient by rank test.

usefulness of C5b-9 and fibronectin in forensic diagnosis of myocardial ischemia through both human samples and animal models ${ }^{15,27-31}$. However, no single immunohistochemical reaction is ideal for diagnosis early myocardial ischemia. The present study demonstrated the usefulness of HO-1 for the postmortem diagnosis of SCD.

HO-1 is a rapidly inducible protein which degrades heme to biliverdin, ferrous iron, and CO in order to protect cells from oxidative stress ${ }^{19-21}$. HO-1 mitigate cellular injury by its anti-oxidant, -apoptotic, and -inflammatory effects ${ }^{21,32-34}$. HO-1-mediated cytoprotection reflects the effects of its catalytic products especially CO because $\mathrm{CO}$ can rescue the injurious effects of HO-1 deficiency ${ }^{21,32-35}$. Although short-term HO-1-mediated cytoprotective effects in heart of animals have been reported ${ }^{36,37}$, it was unknown whether HO- 1 could be an useful indicator of postmortem diagnosis of human AIHD. Novo et al. ${ }^{38}$ determined serum levels of HO-1 in acute MI patients to assess their clinical significance and potential prognostic value. They found that serum HO-1 level was increased in patients of coronary artery diseases, which is in accordance with the cardioprotective role of $\mathrm{HO}-1^{39}$. In the present study, we found that the intranuclear HO-1 expression in cardiomyocytes were detected in $78.2 \%$ (18 cases/23 cases) of AIHD group suggesting that HO-1 expression can be a marker of early ischemia.

In individuals with an ischemic injury for several hours, the myocardial alterations can be detected as follows: necrotic clotting, wavy fibers, hypereosinophilic anucleated myocytes, and early inflammatory infiltration ${ }^{40}$. The contraction bands also have been considered as a sign of ischemia and a histological hallmark of adrenergic stress and/or reperfusion injury ${ }^{41-43}$. In fact, although the frequency was low, about half of AIHD group (12 cases $/ 23$ cases, $52.1 \%$ ) had contraction bands with a significant difference, compared with non-AIHD one (3 cases $/ 33$ cases, $9.0 \%)$.

Activation of inflammation is important to clear the damaged myocardium. Diffuse leukocyte infiltration is first observed after $9 \mathrm{~h}$ from the onset of myocardial ischemia ${ }^{44,45}$. Our data showed few neutrophils and macrophages in the both AIHD and non-AIHD hearts with no significant difference, suggesting that detection of leukocytes in the hearts would not be suited for postmortem diagnosis of early phase of AIHD.

The early loss of myoglobin within $6 \mathrm{~h}$ from the onset of the symptoms is clearly documented in immunohistochemical studies on human samples ${ }^{26,27,46,47}$. This marker has been tested enough sensitive and specific for the detection of AIHD lasting for several hours, however, may not be suitable for detection of very early myocardial ischemic damage.

C5b-9 is most commonly used to support the final diagnosis of SCD because it can reveal areas of myocardial necrosis ${ }^{5,15,27,30}$. This property of C5b-9 is due to its direct involvement in the complement cascade. Indeed, it has been reported that the C5b-9 complex, which is known to form under ischemic conditions, may directly contribute to cardiomyocyte damage ${ }^{48}$. In addition, immunohistochemical demonstrations of fibronectin have been shown to be useful in the localization and diagnosis of early ischemic myocardial necrosis ${ }^{5,15,27,31}$. Fibronectin leaks from damaged capillaries and extracellular matrix to damaged myocytes during ischemia. Hu et al. reported experimentally observing a positive reaction of fibronectin in the cytoplasm of necrotic myocytes 30-60 min after coronary artery occlusion ${ }^{49}$.

For the immunohistochemical evaluation of C5b-9 and fibronectin in the previous studies, positive staining was graded ${ }^{5,15,27,31}$. On the other hand, the interpretation of HO-1 staining in our study is evaluated as positive or negative, which imply that results may be less influenced by each investigator, resulting in less variation of the interpretation. This suggests that our study would be more simple and advantageous in forensic practices, compared with the previous studies.

In this study, we proposed a useful marker HO- 1 in the forensic diagnosis of acute myocardial ischemia. In forensic practices, it is difficult to make definite evaluation of the cause of death, wound ages or wound vitality using a single marker. In line with this, no single immunohistochemical reaction is ideal for diagnosis of early myocardial ischemia in the aspects of forensic safety. Taken together, the combined analysis using several different markers such as HO- 1 as well as C5b-9 and fibronectin could provide more objective and accurate information for the evaluation of early myocardial damage, eventually contributing to the advance of postmortem diagnosis of AIHD when macroscopic or microscopic evidence is insufficient.

\section{Methods}

Antibodies (Abs). The following polyclonal or monoclonal Abs (pAbs or mAbs) were used for immunohistochemical analyses in the present study: goat anti-human HO-1 pAbs (1:200, \#ADI-SPA-896, Enzo Life Science, Farmingdale, NY), rabbit anti-myeloperoxidase (MPO) pAbs (1:100, RB-373-A, Lab Vision/Neo Markers, Fre- 


\begin{tabular}{|c|c|c|c|c|c|c|}
\hline \multirow[b]{2}{*}{ Cause of death } & \multirow[b]{2}{*}{ No } & \multirow[b]{2}{*}{ Male/female } & \multicolumn{2}{|c|}{ Age (years) } & \multicolumn{2}{|c|}{ PMI (h) } \\
\hline & & & Range & Mean & Range & Mean \\
\hline AIHD & 23 & $18 / 5$ & $29-100$ & 71.8 & 7-96 & 27.2 \\
\hline Drowning & 18 & $11 / 7$ & $39-93$ & 73 & $10-48$ & 26 \\
\hline Asphyxia & 3 & $1 / 2$ & $37-54$ & 47.6 & $12-36$ & 20.6 \\
\hline Fire death & 3 & $2 / 1$ & $62-90$ & 76.6 & $12-24$ & 17.3 \\
\hline Hanging & 2 & $2 / 0$ & $49-52$ & 50.5 & $36-48$ & 42 \\
\hline Acute drug poisoning & 3 & $2 / 1$ & $30-44$ & 36 & $24-48$ & 36 \\
\hline Acute carbon poisoning & 1 & $1 / 0$ & 48 & 48 & 30 & 30 \\
\hline Traumatic shock & 1 & $0 / 1$ & 43 & 43 & 12 & 12 \\
\hline Sepsis & 1 & $0 / 1$ & 50 & 50 & 30 & 30 \\
\hline Severe head injury & 1 & $1 / 0$ & 59 & 59 & 72 & 72 \\
\hline Total & 56 & $38 / 18$ & $29-100$ & 67.6 & $7-96$ & 27.5 \\
\hline
\end{tabular}

Table 1. Forensic autopsy cases.

mont, CA), mouse anti-human macrophage marker (CD68) mAb (1:100, clone MAC387, sc-66204, Santa Cruz, Dallas, TX), rabbit anti-human myoglobin pAbs (1:2000, \#A0324, DAKO, Santa Clara, CA).

Human myocardial tissues. A total of 56 human forensic autopsy cases (38 males and 18 females) with a postmortem interval (PMI) of less than $96 \mathrm{~h}$ were selected based on autopsy documents. The individual ages ranged from 29 to 100 years (mean age, 67.6 years). In each case, the cause of death was carefully diagnosed on the basis of complete autopsy, histology, toxicology, and diatom test. Cases were divided into two groups as follows: 23 acute ischemic heart disease with advanced sclerosis and/or stenosis in coronary artery and 33 others including drowning (18 cases), asphyxia (3 case), fire death (3 cases), hanging ( 2 cases), acute drug poisoning ( 3 cases), and one each of acute carbon monoxide poisoning, traumatic shock, sepsis, severe head injury. The detail profiles of all cases (gender, age, PMI) are shown in Table 1.

Histopathological analyses. Heart samples were fixed in $4 \%$ formaldehyde buffered with PBS and then embedded with paraffin. Sections were stained with hematoxylin-eosin (HE) and Masson trichrome for histological analysis. Immunohistochemical analysis was also performed using anti-HO-1, -MPO, -Macrophage Marker, or -myoglobin Abs. Deparaffinized sections were immersed in $0.3 \% \mathrm{H}_{2} \mathrm{O}_{2}$ in methanol for 30 min to eliminate endogenous peroxidase activities. The sections were further incubated with PBS containing $1 \%$ normal serum corresponding to the secondary Abs and $1 \% \mathrm{BSA}$ to reduce nonspecific reactions. The sections were incubated with primary Abs at $4{ }^{\circ} \mathrm{C}$ overnight. Normal rabbit, goat, or mouse IgG was used as negative control. After incubation with biotinylated secondary Abs, immune complexes were visualized using the Catalyzed Signal Amplification System (Dako Cytomation, Kyoto, Japan) according to the manufacturer's instructions.

Morphometrical analysis. Morphometrical analysis was performed with immunohistochemical findings. Cases in which HO-1-positive cells were uniformly observed in myocardial samples were defined as HO- $1^{+}$ cases. These analyses were performed by two investigators with no prior knowledge of the samples.

Statistical analysis. Comparison of two groups was performed using two-side unpaired Student's $t$ test to identify significant difference. $P<0.05$ was considered as significant. All statistical analyses were performed using Statcel3 software under the supervision of a medical statistician.

\section{Data availability}

The authors declare that all data are available in the article file, or available from the authors upon reasonable request.

Received: 6 April 2021; Accepted: 22 October 2021

Published online: 08 November 2021

\section{References}

1. Corrado, D., Zorzi, A., Vanoli, E. \& Gronda, E. Current challenges in sudden cardiac death prevention. Heart Fail. Rev. 25(1), 99-106. https://doi.org/10.1007/s10741-019-09830-0 (2020).

2. Hayashi, M., Shimizu, W. \& Albert, C. M. The spectrum of epidemiology underlying sudden cardiac death. Circ. Res. 116(12), 1887-1906. https://doi.org/10.1161/CIRCRESAHA.116.304521 (2015).

3. Jennings, R. B. \& Ganote, C. E. Structural changes in myocardium during acute ischemia. Circ. Res. 35(Suppl 3), 156-172 (1974).

4. Aljakna, A., Fracasso, T. \& Sabatasso, S. Molecular tissue changes in early myocardial ischemia: From pathophysiology to the identification of new diagnostic markers. Int. J. Legal Med. 132(2), 425-438. https://doi.org/10.1007/s00414-017-1750-z (2018).

5. Sabatasso, S. et al. Early markers for myocardial ischemia and sudden cardiac death. Int. J. Legal Med. 130(5), 1265-1280. https:// doi.org/10.1007/s00414-016-1401-9 (2016).

6. Lie, J. T., Holley, K. E., Kampa, W. R. \& Titus, J. L. New histochemical method for morphologic diagnosis of early stages of myocardial ischemia. Mayo Clin. Proc. 46(5), 319-327 (1971). 
7. Fishbein, M. C. et al. Early phase acute myocardial infarct size quantification: Validation of the triphenyl tetrazolium chloride tissue enzyme staining technique. Am. Heart J. 101(5), 593-600. https://doi.org/10.1016/0002-8703(81)90226-x (1981).

8. Derias, N. W. \& Adams, C. W. Macroscopic enzyme histochemistry in myocardial infarction: Artefactual nature of the creatine phosphokinase reaction. J. Clin. Pathol. 35(4), 407-409. https://doi.org/10.1136/jcp.35.4.407 (1982).

9. Caulfield, J. \& Klionsky, B. Myocardial ischemia and early infarction: An electron microscopic study. Am. J. Pathol. 35(3), 489-523 (1959).

10. Hayakawa, B. N., Jorgensen, A. O., Gotlieb, A. I., Zhao, M. S. \& Liew, C. C. Immunofluorescent microscopy for the identification of human necrotic myocardium. Arch. Pathol. Lab. Med. 108(4), 284-286 (1984).

11. Vargas, S. O., Sampson, B. A. \& Schoen, F. J. Pathologic detection of early myocardial infarction: A critical review of the evolution and usefulness of modern techniques. Mod. Pathol. 12(6), 635-645 (1999).

12. Bardales, R. H., Hailey, L. S., Xie, S. S., Schaefer, R. F. \& Hsu, S. M. In situ apoptosis assay for the detection of early acute myocardial infarction. Am. J. Pathol. 149(3), 821-829 (1996).

13. Knight, B. A further evaluation of the reliability of the HBFP stain in demonstrating myocardial damage. Forensic Sci. Int. 13(3), 179-181. https://doi.org/10.1016/0379-0738(79)90285-8 (1979).

14. Leadbeatter, S., Wawman, H. M. \& Jasani, B. Immunocytochemical diagnosis of early myocardial ischaemic/hypoxic damage. Forensic Sci. Int. 40(2), 171-180. https://doi.org/10.1016/0379-0738(89)90144-8 (1989).

15. Campobasso, C. P. et al. Sudden cardiac death and myocardial ischemia indicators: A comparative study of four immunohistochemical markers. Am. J. Forensic Med. Pathol. 29(2), 154-161. https://doi.org/10.1097/PAF.0b013e318177eab7 (2008).

16. Fracasso, T., Karger, B., Pfeiffer, H., Sauerland, C. \& Schmeling, A. Immunohistochemical identification of prevalent right ventricular ischemia causing right heart failure in cases of pulmonary fat embolism. Int. J. Legal Med. 124(6), 537-542. https://doi. org/10.1007/s00414-009-0382-3 (2010).

17. Fracasso, T., Pfeiffer, H., Sauerland, C. \& Schmeling, A. Morphological identification of right ventricular failure in cases of fatal pulmonary thromboembolism. Int. J. Legal Med. 125(1), 45-50. https://doi.org/10.1007/s00414-010-0486-9 (2011).

18. Bi, H. et al. Immunohistochemical detection of S100A1 in the postmortem diagnosis of acute myocardial infarction. Diagn. Pathol. 8, 84. https://doi.org/10.1186/1746-1596-8-84 (2013).

19. Czibik, G., Derumeaux, G., Sawaki, D., Valen, G. \& Motterlini, R. Heme oxygenase-1: An emerging therapeutic target to curb cardiac pathology. Basic Res. Cardiol. 109(6), 450. https://doi.org/10.1007/s00395-014-0450-9 (2014).

20. Morse, D. \& Choi, A. M. K. Heme oxygenase-1: The "emerging molecule" has arrived. Am J Respir Cell Mol Biol. 27(1), 8-16. https:// doi.org/10.1165/ajrcmb.27.1.4862 (2002).

21. Otterbein, L. E. \& Choi, A. M. Heme oxygenase: Colors of defense against cellular stress. Am. J. Physiol. Lung Cell Mol. Physiol. 279(6), L1029-L1037. https://doi.org/10.1152/ajplung.2000.279.6.L1029 (2000).

22. Abraham, N. G. \& Kappas, A. Pharmacological and clinical aspects of heme oxygenase. Pharmacol. Rev. 60(1), 79-127. https:// doi.org/10.1124/pr.107.07104 (2008).

23. Stocker, R., Yamamoto, Y., McDonagh, A. F., Glazer A. N. \& Ames B. N. Bilirubin is an antioxidant of possible physiological importance. Science. 235(4792), 1043-1046. https://doi.org/10.1126/science.3029864 (1987).

24. Motterlini, R. \& Otterbein L. E. The therapeutic potential of carbon monoxide. Nat. Rev. Drug Discov. 9(9), 728-743. https://doi. org $/ 10.1038 / \mathrm{nrd} 3228(2010)$.

25. Biswas, C. et al. Nuclear heme oxygenase-1 (HO-1) modulates subcellular distribution and activation of Nrf2, impacting metabolic and anti-oxidant defenses. J. Biol. Chem. 289(39), 26882-26894. https://doi.org/10.1074/jbc.M114.567685 (2014).

26. Brinkmann, B., Sepulchre, M. A. \& Fechner, G. The application of selected histochemical and immunohistochemical markers and procedures to the diagnosis of early myocardial damage. Int. J. Legal Med. 106(3), 135-141. https://doi.org/10.1007/BF01225234 (1993).

27. Ortmann, C., Pfeiffer, H. \& Brinkmann, B. A comparative study on the immunohistochemical detection of early myocardial damage. Int. J. Legal Med. 113(4), 215-220. https://doi.org/10.1007/s004149900094 (2000).

28. Casscells, W., Kimura, H., Sanchez, J. A., Yu, Z. X. \& Ferrans V. J. Immunohistochemical study of fibronectin in experimental myocardial infarction. Am. J. Pathol. 137(4), 801-810 (1990).

29. Thomsen, H. \& Held, H. Immunohistochemical detection of C5b-9(m) in myocardium: An aid in distinguishing infarction-induced ischemic heart muscle necrosis from other forms of lethal myocardial injury. Forensic Sci. Int. 71(2), 87-95. https://doi.org/10. 1016/0379-0738(94)01640-q (1995).

30. Schäfer, H., Mathey, D., Hugo, F. \& Bhakdi, S. Deposition of the terminal C5b-9 complement complex in infarcted areas of human myocardium. J. Immunol. 137(6), 1945-1949 (1986).

31. Hu, B. J., Chen, Y. C. \& Zhu, J. Z. Study on the specificity of fibronectin for post-mortem diagnosis of early myocardial infarction. Med. Sci. Law 42(3), 195-199. https://doi.org/10.1177/002580240204200303 (2002).

32. Abraham, N. G. \& Kappas, A. Heme oxygenase and the cardiovascular-renal system. Free Radic. Biol. Med. 39(1), 1-25. https:// doi.org/10.1016/j.freeradbiomed.2005.03.010 (2005).

33. Brouard, S., Otterbein, L. E., Anrather, J., Tobiasch, E., Bach, F. H., Choi, A. M. \& Soares, M. P. Carbon monoxide generated by heme oxygenase 1 suppresses endothelial cell apoptosis. J. Exp. Med. 192(7), 1015-1026. https://doi.org/10.1084/jem.192.7.1015 (2000).

34. Lee, T. S. \& Chau, L. Y. Heme oxygenase-1 mediates the anti-inflammatory effect of interleukin-10 in mice. Nat. Med. 8(3), 240-246. https://doi.org/10.1038/nm0302-240 (2002).

35. Sato, K. et al. Carbon monoxide generated by heme oxygenase-1 suppresses the rejection of mouse-to-rat cardiac transplants. J. Immunol. 166(6), 4185-4194. https://doi.org/10.4049/jimmunol.166.6.4185 (2001).

36. Yoshida, T., Maulik, N., Ho, Y. S., Alam, J. \& Das, D. K. $\mathrm{H}_{\text {mox- } 1}$ constitutes an adaptive response to effect antioxidant cardioprotection: A study with transgenic mice heterozygous for targeted disruption of the Heme oxygenase-1 gene. Circulation 103(12), 1695-1701. https://doi.org/10.1161/01.cir.103.12.1695 (2001).

37. Yet, S. F. et al. Cardiac-specific expression of heme oxygenase-1 protects against ischemia and reperfusion injury in transgenic mice. Circ. Res. 89(2), 168-173. https://doi.org/10.1161/hh1401.093314 (2001).

38. Novo, G. et al. Hsp60 and heme oxygenase-1 (Hsp32) in acute myocardial infarction. Transl. Res. 157(5), 285-292. https://doi. org/10.1016/j.trsl.2011.01.003 (2011).

39. Wang, G. et al. Cardioprotective and antiapoptotic effects of heme oxygenase-1 in the failing heart. Circulation 121(17), 1912-1925. https://doi.org/10.1161/CIRCULATIONAHA.109.905471 (2010).

40. Baroldi, G., Mittleman, R. E., Parolini, M, Silver, M. D. \& Fineschi, V. Myocardial contraction bands. Definition, quantification and significance in forensic pathology. Int. J. Legal Med. 115(3), 142-151. https://doi.org/10.1007/s004140100229 (2001).

41. Fineschi, V. Measuring myocyte oxidative stress and targeting cytokines to evaluate inflammatory response and cardiac repair after myocardial infarction. Curr. Vasc. Pharmacol. 13(1), 3-5. https://doi.org/10.2174/157016111301150303121746 (2015).

42. Turillazzi, E. et al. The meaning of different forms of structural myocardial injury, immune response and timing of infarct necrosis and cardiac repair. Curr. Vasc. Pharmacol. 13(1), 6-19. https://doi.org/10.2174/15701611113119990008 (2015).

43. Fishbein, M. C., Maclean, D. \& Maroko, P. R. The histopathologic evolution of myocardial infarction. Chest 73(6), 843-849. https:// doi.org/10.1378/chest.73.6.843 (1978).

44. Markwerth, P., Bajanowski, T., Tzimas, I. \& Dettmeyer, R. Sudden cardiac death-update. Int. J. Legal. Med. 1-13. https://doi.org/ 10.1007/s00414-020-02481-z (2020). 
45. Dettmeyer, R. B. Forensic Histopathology, Fundamentals and Perspectives, Second edition. Printforce, the Netherlands, 303-355

46. Hansen, S. H. \& Rossen, K. Evaluation of cardiac troponin I immunoreaction in autopsy hearts: A possible marker of early myocardial infarction. Forensic Sci. Int. 99(3), 189-196. https://doi.org/10.1016/s0379-0738(98)00193-5 (1999).

47. Jasra, S. K., Badian, C., Macri, I. \& Ra, P. Recognition of early myocardial infarction by immunohistochemical staining with cardiac troponin-I and complement C9. J. Forensic Sci. 57(6), 1595-1600. https://doi.org/10.1111/j.1556-4029.2012.02172.x (2012).

48. Homeister, J. W., Satoh, P. \& Lucchesi, B. R. Effects of complement activation in the isolated heart. Role of the terminal complement components. Circ. Res. 71(2), 303-319. https://doi.org/10.1161/01.res.71.2.303 (1992).

49. Hu, B. J., Chen, Y. C. \& Zhu, J. Z. Immunohistochemical study of fibronectin for postmortem diagnosis of early myocardial infarction. Forensic Sci. Int. 78(3), 209-217. https://doi.org/10.1016/0379-0738(96)01891-9 (1996).

\title{
Acknowledgements
}

We sincerely thank Ms. Emi Shimada for her technical assistance. This work was supported by Japan Society for the Promotion of Science, Grant-in-Aid Scientific Research (B) No. JP20H03957 (Y. I.) and Challenging Research (Exploratory) No. JP18K19.694 (T. K.).

\section{Author contributions}

Conceptualization: Y.I., T.K.; Formal Analysis: Y.K., Y.I., M.N., A.I., A.T., E.S., H.Y. and M.O.; Funding Acquisition: Y.K., Y.I. and T.K; Investigation: Y.K. and Y.I.; Project Administration: T.K.; Validation: M.N. and A.K.; Writing-Original Draft Preparation: Y.I.; Writing_Review and Editing: F.F. and T.K.

\section{Competing interests}

The authors declare no competing interests.

\section{Ethical approval}

Our study was approved by the Research Ethics Committee of Wakayama Medical University (No. 3176). All procedures were carried out in accordance with the Declaration of Helsinki Principles. Moreover, this study was conducted using autopsy records from the past, and we could not obtain informed consent from the bereaved family for the use of the records. Therefore, in accordance with the "Ethical Guidelines for Medical Research Involving Human Subjects (enacted by the Ministry of Health, Labor and Welfare in Japan)," Sect. 12-1 (2) (a) (c). Thus, the review board of Research Ethics Committee of Wakayama Medical University waived the need for written informed consent from relatives of individuals studied since this was a de-identified retrospective study of archived autopsy-derived tissue.

\section{Additional information}

Supplementary Information The online version contains supplementary material available at https://doi.org/ 10.1038/s41598-021-01102-y.

Correspondence and requests for materials should be addressed to Y.I. or T.K.

Reprints and permissions information is available at www.nature.com/reprints.

Publisher's note Springer Nature remains neutral with regard to jurisdictional claims in published maps and institutional affiliations.

\begin{abstract}
(c) (1) Open Access This article is licensed under a Creative Commons Attribution 4.0 International License, which permits use, sharing, adaptation, distribution and reproduction in any medium or format, as long as you give appropriate credit to the original author(s) and the source, provide a link to the Creative Commons licence, and indicate if changes were made. The images or other third party material in this article are included in the article's Creative Commons licence, unless indicated otherwise in a credit line to the material. If material is not included in the article's Creative Commons licence and your intended use is not permitted by statutory regulation or exceeds the permitted use, you will need to obtain permission directly from the copyright holder. To view a copy of this licence, visit http://creativecommons.org/licenses/by/4.0/.
\end{abstract}

(c) The Author(s) 2021 